\title{
TWO CASES
}

\section{or \\ A N E U R I S M,}

IN WHICE

THE TEMPORARY LIGATURE WAS EMPLOYED.

Bx B. TRAVERS, EsQ. F.R.S.

VICE-PRESIDENT OF THE SOCIETY.

Read May 26, 1818.

THE following are the particulars of two cases, in which I removed the ligature at different periods after the operation for Aneurism.

\section{CASE I.}

John Smith, labourer, aged thirty-seven, was admitted into St. Thomas's Hospital in the beginning of February 1817. He had a tense diffused tumor in the bend of the right elbow, inclining towards the inner condyle; it was about the size of half a hen's egg, giving no pain when handled, and free from pulsation. The brachial artery.could 
be traced on its outer side, passing towards the radius; no pulse wa perceptible in the ulnar artery. The motion of the joint was not at all impeded, but all the fingers and thumb, the little finger excepted, were benumbed, and he had nearly lost the power of extending or bending them.

He stated that about eight months previous to his admission he was bled; the cicatrix could be seen on the inner and. lower part of the tumor, about an inch distant from the artery. Soon after the bandage was applied, the arm became generally swollen as far as the wrist; but by degrees, and under the application of lotions, the swelling of the arm subsided, and became more circumscribed around the orifice, pulsating strongly and distinctly, and continuing to do so until within a few days of his application at the Hospital.

February 14th. The tumor being considered aneurismal, the brachial *artery was tied an inch and a half above the bend of the elbow with a noose ligature, so as to render its removal practicable if judged expedient. The pulsation in the radial artery immediately ceased.

15th. He had suffered considerable pain, which completely deprived him of rest during the night, but was this morning quite easy, and stated that the pain and numbness in the hand had consi- 
derably abated; the radial artery pulsated, but with diminished force; in the ulnar no pulsation was perceptible; the arm was of the natural temperature ; pulse about 70.' At about six o'clock in the evening he arose suddenly from his' bed, and stated a determination to quit the Hospital and return home, at the same time soliciting the removal of the ligature. He talked incoherently respecting his family, and became very turbulent towards night. Pulse 120; bowels had not been moved; he took accordingly a purgative draught.

The person who accompanied this man to town stated, that previously to this attack he had occasionally been the subject of mental derangement, insomuch that coercive measures had been employed to restrain him.

16th. He had spent a night of incessant restlessness, and could with difficulty be kept in bed. He now took a dose of castor oil, and shortly had a copious evacuation of a healthy character, after which he became more composed. Pulse about 100. In the afternoon he was tranquil and collected; at four; the ligature was removed with little difficulty, after having remained on the artery.for fifty hours. The wound had adhered, except around the ligature, which was imbedded in coagulable lymph. In the evening he was again refractory and noisy; had another full 
motion.; no pulsation in the artery below the portion which had been tied.

17th. During the night he enjoyed about three hours' sound sleep. His brother visited him in the morning, since which he had been more composed. Pulse natural; tongue perfectly clean; a small healthy discharge of pus issued from' $h$ opening left by the ligature; the pulsation in the radial artery was feeble; nor could any be perceived either in the brachial immediately above the tumor, or in the ulnar artery.

18th. Had slept the whole of the night, and was perfectly tranquil and collected; took a dose of castor oil, which operated briskly; pulse was now 76, soft and free; arm easy.

19th, 20th, and 21st. He continued improving; pulse-was natural; no pulsation could be felt for some space above the part where the ligature had been applied; wound appeared healthy and free from discharge; the tumor became slightly discoloured and tumefied, but continued free from pain; the white wash was applied. On the last of these days, after having spent rather a restless night, a slight inflammatory blush was seen surrounding the wound, which now discharged a small quantity of matter. On the $22 \mathrm{~d}$ he had a slight recurrence of his mental 
disorder; the wound discharged but little, and had rather a languid aspect; continued taking the aperient.

March 1st. The wound was now completely closed by granulations, which were rather spongy; a lotion of sulphate of zinc was applied; the tumor became less tense and more oircumscribed; there was still a degree of numbness and pain in the hand; the pulsation in the radial artery was not so strong or so firm as before the operation, and in the ulnar was not at all perceptible; the artery was filled by a coagulum for some. space above the wound, and below it a very faint thrilling sensation was conveyed to the fingers.

From the 1st to the 12th he continued improv. ing daily; there was a slight disposition in the cicatrix to ulcerate, and the sac remained in the same state. He quitted the Hospital on the 14th of March of his own accord, and it was afterwards ascertained, that within three months from his removal he had fought two sharply contested battles, being a noted pugilist in his county.

CASE II.

William Edgecombe, lighterman, aged twentytwo, was admitted into St. Thomas's Hospital on VoL. IX. E E 
the $22 \mathrm{~d}$ of November. 1817. This man stated that he had been much employed in carrying heavy loads up steps and ladders. About three weeks previous to his admission, while stepping from one barge to another, he was attacked with pain in the right ham, and three or four days afterwards he perceived a swelling, but never observed a pulse in it until a day or. two before his application at the Hospital. On examination, an aneurism of the popliteal artery, was evident, and he suffered, much pain both at the part and down the leg. His health was perfectly good, and he was free from other complaint.

28th. The operation was performed at one o'clock, and the artery was tied at the usual place with a noose ligature, so as to permit its removal.

Nine P.M. Inclined to sleep, which he had been prevented. doing for a length of time in consequence of the pain; pulse 84 and full; limb equal in warmth to the opposite.

29th, one P.M. He had been restless during the early part of the night, but dozed since three o'clock; pulse 92 and 'soft; leg and foot of natural warmth and free from pain: Sol. Magn. Sulph. ad sedem.

Four P.M. The ligature was removed without 
difficulty after an interval of twenty-seven hours ; no pulsation could be felt in the sac.

Seven P.M. A faint pulsation was clearly perceived in the sac.

30th. Had some pain during the night, but became easy and composed towards morning; pulsation, though very distinct, was less strong than before the operation.

December 1st. He still complained of pain, though to a much less degree than prior to the operation.

2d. Pulsation continued ; he was otherwise doing well.

3rd. He had passed a good night; quite free from pain; pulsation much the same.

6th. The wound was dressed for the fourth time; its edges were covered with healthy granulations, and the purulent discharge was in small quantity; pulsation the same; no recurrence of pain. Pressure was now applied by means of a roller from below the knee to the groin, and continued for the space of a month without inconvenience to the patient, during which time the pulsation in the sac evidently became feebler. 
Jan. 10th. After sitting up in bis bed on Wednesday the 7 th, he felt a pain in the tumor, which con. tinued increasing and at length became very severe; tumor very tense, and no pulsation coald now be felt in it. Eight P.M. Pain was excessive; pulse quick, full, and hard; skin hot; sixteen ounces of blood were taken from his arm, and he took forty drops of Tinct. Opii.

Jan. 11th. The pain ceased entirely at one A.M. but returned at nine, and continued, though in a less degree; pulse quick and full; perspiration free and general; tongue rather furred; the tumor was more diffused and less prominent.

Three P.M. Pain increased; pulse 120; the blood drawn yesterday was sizy and cupped; bleeding, repeated : Ol. Ricini $\bar{j}$.

Seven P.M. Less pain since the bleeding; pulse about 110 and softer; bowels freely open.

12th. No increase of pain; pulse 108. At about two in the afternoon the pain increased, when ten ounces of blood were taken from him; this blood was a hitle cupped; pulse about 96, of moderate fulness; pain very severe; no alteration in the tumor. At two o'clock he was conveyed to the Operating Theatre, and the artery was again tied about two inches above the place 
at which the former ligature had been applied. In the evening he was free from pain in the sac; limb was also warm.

13th. He had passed a comfortable night, and was free from pain; the leg was of a natural tem. perature; had had two motions previous to the operation, but none since: Sol. Magn. Sulph.

14th. He was cheerful and perfectly easy; the sac was diminished in bulk and more flaccid; limb warm; he had no recurrence of pain; the old wound was completely healed, but the recent one seemed disposed to ulcerate; he now partook of the house diet, and drank a pint of porter daily.

24th. The ligature came away with the dressing (12th day); wound still continued languid, though improving.

In a day or two after, Feb. 7th, he complained of pain in the right hypochondrium and at the scapula, and being feverish was seen by the physician. The wound continued improving until the 18th, when it was perfectly healed, and on the 26th he was discharged cured, with the usual degrae of lameness. 


\section{Observations.}

The case of Smith satisfactorily shews, that the residence of the ligature upon the artery for a period of fifty hours as certainly and completely answers the purpose of its application; as if allowed to remain until : thrown off by the natural process.

In considering the case of Edgecombe, I think it evident, from the suspension of the pain and the diminished strength of the pulsation for a month following the application of the temporary ligature, that it had occasioned a degree of impediment to the current of blood in the artery; and these circumstances led me at one time to entertain a hope that the cure of the Aneurism was gradually accomplishing. But this was fallacious; and I may observe, that no such expectation ought ever to be entertained while the pulsation in any degree of the aneurismal sac continues. For the establishment of a collateral circulation is insufficient for the cure of the Aneurism, while the sac continues to receive a full jet of blood. I have seen a case in which the artery being obliterated below the sac, collateral circulation was established; yet the pulsation of the sac was undiminished and the progress of the disease unretarded. On the other hand, the arrestation of the pulse in the sac, unless attended by a diminution of its volume 
and freedom from pain, affords no just ground for the opinion that the natural cure of the Aneurism is in progress; and if it be accompanied by an increase of the tumor, an aggravation of pain and the symptoms of inflammatory action, the reverse must be our conclusion : the operation is the only alternative, and cannot with safety be delayed. These symptoms are in fact those which occur where no operation has been attempted, and depend on the further yielding of the sac and surrounding parts. Let me repeat, that non-pulsation of the sac is a sign auspicious or otherwise, simply as it stands connected with increase or diminution of bulk and pain.

It is scarcely necessary to add to the narration of this case, my determination to relinquish the use of the temporary ligature. " I should regret to hear of a repetition of the experiment, being unable upon reflection to discover a single circumstance tending in any degree to impeach its accuracy. But I am far from feeling regret that a practice has been fairly tried, of which $I$ âm free to confess $I$ had formed too favourable an opinion; on the contrary, it is to me a source of high satisfaction, that the decision of the question, which I deemed of importance, has been obtained without loss of life or limb. The grounds of my expectation, and the motives which led me to institute the practice, will be seen by a reference to my papers in the fourth and sixth volumes of the 
Socieiy's Transactions; and it remains only that $I$ should add one or two inferences from the above cases, before finally closing the subject.

1st. It appears that a ligature upon the divided carotid of the horse applied for twelve hours, moy be removed without hemorrhage*; whereas the removal of a ligature upon the femoral artery of the human subject in twenty-seven hours, is followed by the return of the circulation in the vessel.

2dly. That although a ligature upon the carotid of a horse applied for six hours, has in a period of seventy hours been followed by a complete obstruction of its canalt; a ligature applied for twentyseven hours upon the human femoral artery, has scarcely afforded a perceptible impediment to the blood. Is this variation to be referred to a difference in strength or susceptibility of inflammation? or to the influence of mind upon the circulation? Does the comparative slowness of the circulation in the horse, or the difference in the force and direction of the current in the carotid and femoral arteries explain it?

3rdly. The adhesive union is prevented by the inclosure of a foreign body in a wound, long before suppuration has commenced. Suppuration is

* See Med. Chir. Trans. Vol. VI. p. 637, 640.

t See Mod. Chir. Trans. Vol. VI. p. 647. 
as certain to take place, though the ligature removed after a few hours, as if it were left to be cast off; and the granulating process is languidly performed after an interruption in its early stage, for the purpose of removing the obstacke to union, than where no such interrup. tion has been given, and the obstacle has been removed by nature's own means. Hence it fol, lows, that the theory, which, in removing the ligature within a given time, proposed the double advantage of a quicker as well as a surer pro cess, fails in both points when brought to the test of practice upon the human subject. I might add for the sake of companison a third case, in which I left the ligatire to nature, and it was cast off on the 19th day, in the highest degree satisfactory. But this operation is sufficiertly established and appreciated, and I am disposed to believe that, when properly perfermed, it ad. mits of no improvement.

Since I had the honour of presenting these cases to the Society, Professor Scarpa has publiohed a memoir on the ligature of the principal arteries of the extremities. In a series of experiments on the sheep, the dog and the cow, he ascertained that the carotid artery, compressed by a cylindrical roll of plaster under a flat ligature, was completely obstructed on the fourth day, its coats remaining entire. He relates four cases in which 
the ligature thus applied to the arteries of the human subject was: removed on the fourth day with success.

The following statement is extracted from the report of a conversation between the Professor and the Editors of the Journal of Foreign Medicine and Surgery*.

" He said that he still continued to recommend a cylinder of waxed linen to be placed between the artery and the ligature, and that he had never once met with a secondary hemorrhagy since he began to employ this contrivance. He always made use of two ligatures, but in a manner, he 'said, which had none of the dangers of the ligature d'attente. During the operation, he was very cautious to separate the artery from the surrounding cellular substance to an exceedingly small extent, and having thus introduced the two ligatures under the artery, he tied one of them over the cylinder of linen, leaving the other not tied." From his experiments, "he had been led to conclude, that in the human subject the ligature might with safety, and with success to the operation, be removed on the fourth day. He would not however remove, he said, both the ligatures at that time. He would leave the one which had

* Quarterly Journal of Foreign Medicine and Surgery, No. I. page 80. 
not been tied, in order that if the pulsation returned in the tumor, the artery might again be tied -without any second operation. He then stated, at some length, the advantages of this early removal of the ligature over the present plan of leaving it perhaps fourteen days, insisting chiefly on the almost certain avoidance of secondary hemorrhagy, on the avoidance of a tedious suppuration in the wound, and occasionally of a troublesome sinus.

“6 He said, that he considered this improvement in the operation for Aneurism as more valuable than all the bagatelles, as he was pleased to call them, which he had ever made."

The professional science and character of Scarpa' demand that every suggestion of his experience should be received with respectful deference. But the view which the surgeons of this country have been led to take of the operation of the ligature, must undergo a total change before they can acquiesce in his statement of the superior advantages of the method above described. It has been long well known that pressure will obliterate the canal of an artery, and in a late series of experiments $I$ endeavoured to shew its efficacy in comparison with the ligature.

All the advantage which I proposed in removing the ligature, was the union of the wound by the direct adhesive inflammation, and of course 
connected with this, the avoidance of sinuses and secondary hemorrhage. But finding that the removal of the ligature in a shorter time than admitted of its successful operation, would not procure this advantage; that the wound would still suppurate at the place of the ligature, and small as it was, would heal tediously; I have abandoned it, as failing of the point proposed.

The greater detachment of the vessel required for the introduction of two flat ligatures and a foreign body, than for the passage of a single round ligature, cannot be denied; therefore the good obtained by the removal of these substances, when the suppuration is completely established, as on the fourth day, can only be admitted as regards the practice of employing them*, which is objectionable, as it renders the suppuration more extensive, and the wound slower in healing, than if but one round ligature had been employed. But the practice of placing extranoous bodies in wounds, and, in general, of complicating operations, is so adverse to the genius of English surgery, that it is unnecessary to dwell upon these points; and indeed I may add, that the success of the operetion as now generally performed in this country,

* I have been long in the occasional practice of applying the noose ligatures to the arteries of stumps, and removing them all at the first dressing. I have not known a single instance of secondary hemorrhage. This fact excited much surprise when I lately mentioned it to some Parisian surgeons of cminence. 
seems to render all contrivances superfluous for the prevention of secondary hemorrhage.

In our appeal to experience, it should be recollected that the favourable execution of an ill-concerted operation affords a better chance of success than the mal-adroit execution of a good one. This and the difficulty or impossibility of obtaining an impartial history of what are called successful cases, render the decisions of mere experience somewhat equivocal. In a seeming equipoise of facts, we must call in the aid of theory to correct the balance and decide our judgments. Without this aid it would be exceedingly difficult to determine the eligibility of one operation over another, as, for example, not to speak of the broad and round ligature upon arteries, the different operations for stone, cataract, aneurism, \&c. And although it is vain to hope that such points will ever cease to be the subjects of controversy, yet a conformity to certain acknowledged principles of science will always decide the question of preference with the majority of intelligent practitioners. 